\title{
Effect of Genomic and Amino Acid Sequence Mutation on Virulence and Therapeutic Target of Severe Acute Respiratory Syndrome Coronavirus-2 (SARS COV-2)
}

\author{
Endriyas Kelta Wabalo \\ Abebe Dukessa Dubiwak \\ Mengistu Welde Senbetu (D) \\ Tariku Sime Gizaw (D) \\ Department of Biomedical Sciences, \\ Faculty of Medical Sciences, Institute of \\ Health, Jimma University, Jimma, Ethiopia
}

Correspondence: Endriyas Kelta Wabalo Department of Biomedical Sciences, Faculty of Medical Sciences, Institute of Health, Jimma University, Jimma, Ethiopia Email keltatona@gmail.com

\begin{abstract}
The COVID-19 pandemic is caused by severe acute respiratory syndrome coronavirus-2 (SARS-CoV-2). It is one of the RNA coronaviruses which share the highest mutation rates of RNA viruses when compared with that of their hosts. The collective mutation rate of RNA viruses is up to a million times higher than their hosts and is correlated with enhanced virulence of viruses. The RNA, genomic material of SARS-CoV-2, has the capacity of showing amplified fast changes as the infection spreads. These changes were frequently observed in genes for spike glycoprotein, nucleocapsid, ORF1ab, and ORF8, together with RNA dependent RNA polymerase. In contrast, genes for envelope, membrane, ORF6, ORF7a and ORF7b showed no observable changes in terms of amino acid substitutions. Mutated SARS COV-2 at these particular sites has been associated with viral infectivity, false laboratory results and viral genome mutation and interferes with therapeutic targets. Interferences with therapeutic targets is frequently observed in genes for RdRp. Additionally, mutated viral genes for RdRp render slow fidelity of RdRp protein, resulting in a high mutation rate. Such a high mutation rate might allow new virulent forms of the virus to emerge and influence the disease profile. This review aimed to elaborate on the effect of genomic and amino acid sequence mutations on the virulence and therapeutic targets of SARS COV-2. To achieve this objective, multiple literatures have been reviewed.
\end{abstract}

Keywords: genomic mutations, amino acid sequence mutations, virulence, therapeutic target, RdRp, SARS COV-2, spike proteins

\section{Introduction}

\section{Background Information}

Severe acute respiratory syndrome coronavirus-2 (SARS-CoV-2) is an RNA coronavirus belonging to genus $\beta$-coronavirus and a member of the family of Coronaviridae. ${ }^{1}$ Most Coronaviridae families, including those causing respiratory problems in human hosts, are belonging to two genera, $\alpha$-coronavirus and $\beta$-coronavirus. The extent of respiratory problems differs from person to person. For instances, it causes mild infection in healthy adults but has much more severe effects in the elderly and patients with co-morbidities. The later can experience severe damage to their respiratory systems, sometimes dying as a result. ${ }^{2}$ Several literatures indicated that people older than 65 years and those with underlying co-morbidities experience higher mortality rates than former ones. ${ }^{2,5-7,9-11}$ 
Fever, cough, shortness of breath and fatigue are common symptoms of the coronavirus disease (COVID-19), caused by SARS COV-2. ${ }^{3,4}$ As indicated in early data, about $20 \%$ of patients developed severe symptoms of COVID-19 accompanied by shortness of breath and requiring hospitalization, including $5 \%$ who are admitted to intensive care units. Besides, the initial estimate of case fatality rates from COVID-19 was from $3.4 \%$ to $6.6 \%$, which was much extermely lower than the $9.6 \%$ for severe acute respiratory syndrome (SARS) or the $34.3 \%$ for Middle East respiratory syndrome (MERS). ${ }^{5-7}$

Regardless of the fact that the outbreak has dramatically expanded to create a global pandemic, ${ }^{8}$ the COVID19 related morbidity, numbers of active cases, and mortality rates differ from country to country. Many studies from different corners of the world speculating on possible reasons for these variations but have produced no credible results, yet. According to different studies from different corners of the globe, the $4715 \mathrm{~L}$ variant of open reading frame $1 \mathrm{ab}$ (ORF1ab) protein, 614G variant of $\mathrm{S}$ protein and RNA dependent RNA polymerase (RdRp) were the commonest viral proteins affected by mutations among others. ${ }^{12,13}$ One study hypothesized that there is a correlation between mutations at the $4715 \mathrm{~L}$ variant of ORF1ab and $614 \mathrm{G}$ variant of $\mathrm{S}$ protein and fatality rates. ${ }^{12}$ Moreover, mutated RdRp may allow the viruses to escape host immunity and to develop resistance to commonly used antiviral therapies. ${ }^{13}$ Therefore, dealing with the effects of mutation on virulence and therapeutic targets of this virus should be needed to overcome such challenges, hence, the aim of this review was to shed light on the effects of genes and amino acid sequence mutation on virulence and therapeutic targets of SARS COV-2.

\section{SARS COV-2 Genome and Genomic Expression}

SARS-CoV-2 has a single strand RNA genome, which is positive-sensed and about 29,903 $\mathrm{kb}$ nucleotides long, making it as one of the largest genomes among RNA viruses. ${ }^{14}$ These nucleotides are organized into 10 ORFgenes encoding for 28 proteins. Four out of 28 proteins are structural proteins (spike (S), envelope (E), membrane $(\mathrm{M})$, and nucleocapsid (N) proteins), 16 are nonstructural proteins (Nsps) and 8 are accessory proteins. ${ }^{14-16,24}$

The spike (S) glycoprotein is comprised 1273 amino acid residues and is critical for the viral infection. It has $\mathrm{N}$-terminus signal peptide of 1-13 amino acid residues, S1 subunit peptide of 14-685 amino acid residues, and S2 sub unit peptide of 686-1273 amino acid residues. ${ }^{18-20}$

The S1 subunit peptide has N-terminal domain (composed of 14-305 stretch of amino acid residues) and a receptor-binding domain (RBD) (composed of 319-541 stretch of amino acid residues. The S2 subunit peptide has a fusion peptide (FP) (composed of 788-806 stretch of amino acid residues), a heptapeptide repeat sequence 1 (HR1) (composed of 912-984 stretch of amino acid residues), an HR2 (composed of a 1163-1213 stretch of amino acid residues), a transmembrane domain (composed of 1213-1237 stretch of amino acid residues), and a cytoplasmic domain (composed of 1237-1273 stretch of amino acid residues). ${ }^{18}$

The virus to host interaction is initiated when S glycoprotein, through its RBD of S1 subunit peptide, interacts with angiotensin-converting enzyme-2 (ACE-2) receptor on the host cell surface. The RBD-ACE-2 interaction allows not only viral infection, but also enhances rapid human-to-human transmission. ${ }^{19,21}$ Mutation of these sitesdo have effect on viral virulence. ${ }^{22}$

The other site on the viral genome prone to mutation is ORF1ab, encoding for two viral polypeptides (Ppla and Pp1b), which generate 16Nsps after cleavage by chymotrypsin-like (3CL) protease $\left(\mathrm{M}^{\mathrm{pro}}\right)$ and papain-like proteases. ${ }^{19,23,24}$

\section{Gene and Amino Acid Mutations of SARS COV-2}

Gene Mutations

RNA viruses overwhelms their immediate hosts in mutation rates which was highly correlated with enhanced virulence of virus. ${ }^{25}$ The SARS-CoV-2 genome showed increasing fast and frequent changes at the level of genes for $\mathrm{S}, \mathrm{N}, \mathrm{ORF} 1 \mathrm{ab}$ ORF8 proteins as the spread of infection amplified. ${ }^{21,26}$

Study done on six geographic regions by taking SARS$\mathrm{CoV}-2$ as the reference identified a total of 1234 mutations. ${ }^{12}$ Another study done in USA analyzed the rate of accumulated viral gene mutations and identified accumulated mutations on genes for helicase, NSP2, S, NSP3,RdRp, ORF3a, ORF8, and N proteins. ${ }^{17}$

\section{Amino Acid Mutations}

\section{Amino Acid Mutations for Structural Proteins}

The S protein depicted substitutions of D614G, V483A, L5F, Q675H, H655Y, and S939F, with D614G mutation identified as the most common amino acid mutations 
among others. ${ }^{21}$ Similarly, R203K and G204R mutations are commonly identified at the level of $\mathrm{N}$ proteins. ${ }^{21,33}$ There are no mutations identified yet in relation to $\mathrm{M}$ and E proteins. The possible reason might be housekeeping functions of these proteins allowing them to have a greater resistance to mutations. ${ }^{21}$

\section{Amino Acid Mutations for Non-Structural Proteins} ORF3a, N, ORF8, ORF1ab, and $\mathrm{S}$ proteins showed the largest number of non-synonymous mutations, with ORF3a taking the leading position. ${ }^{21,34}$ The Q57H and G251V mutations account for the majority of nonsynonymous mutations identified in ORF3a. Similarly, L84S is a major non-synonymous mutation in ORF8, accompanied by a change in V62L. ${ }^{21}$

Comparable levels of mutation frequencies have been observed at the level of NSP2, NSP3 and RdRp proteins with that of ORF3a, N, ORF8, ORF1ab and S proteins. ${ }^{21}$ The nsp2 (Asp268del) deletion mutation is the most common and widely spread type of non-synonymous mutation across Europe ${ }^{57}$ Like E and $\mathrm{M}$ structural proteins, ORF6, 7a, 7b and 10, NSP7, NSP9, NSP10, and NSP11 proteins showed no significant changes in amino acid residues, yet. $^{21,35}$ The 27-amino-acid deletion in ORF7a SARSCoV-2 reported in Arizona was exceptional. ${ }^{56}$

\section{Impact of ORF I ab Gene Mutation in Laboratory Results and Amino Acid Sequences of Encoded Proteins}

ORF1ab occupies more than $75 \%$ of the SARS COV-2 genome and is identified as the highest mutation frequency region. ${ }^{17,27}$ In Japan, three and twenty-four nucleotide deletion mutations were identified within this region, and in Australia, twenty-nine mis-sense mutations were identified in the ORF1ab polyprotein. ${ }^{23}$

False positive laboratory result, as it permit infected individuals to live with other people and, might have an unfavorable impacts on endeavors to control the spread of the virus. ${ }^{28}$

Currently accepted laboratory test for SARS-CoV-2, the real-time polymerase chain reaction (RT-PCR) test, risks producing inconsistent results. The possible reasons for this inconsistency might be mutations in viral RNA sequences affecting RT-PCR-utilizing primers in different genes; or mutations in the primer and probe target regions; or genetic diversity and rapid evolution of SARS-CoV-2 that have been observed in different studies. ${ }^{29}$
The $14,408 \mathrm{C}>\mathrm{T}(\mathrm{P} 4715 \mathrm{~L})$ and $3037 \mathrm{C}>\mathrm{T}(\mathrm{F} 106 \mathrm{~F})$ variants have been recognized as the two most frequently occurring gene mutations at ORF1ab regions, possibly leading to inconsistent RT-PCR test results. The P4715L variant was causing mutations in the RdRp/NSP12 and responsible for lower replication fidelity of the enzyme. The F106F variant, on the other hand, was causing mutations in the Nsp3 gene. ${ }^{30,31}$

Furthermore, ORF1ab regions of the virus from different geographic regions showed mutation of amino acid sequences of encoded proteins. An isolate from China showed N2708S and F2908I, whereas from South Korea and Sweden showed G818S and F4321L. On the other hand, an isolate from Brazil showed L3606F and from Vietnam showed R3323C. ${ }^{32,58}$

\section{Effect of Mutant Spike Protein on Virulence of SARS COV-2}

The affinity of RBD of the S sub-unit peptide of the $S$ protein for ACE-2 and the overall infectivity of the virus has been potentially altered by gene mutations for spike. ${ }^{23,36}$ The overall impact is either reduced ${ }^{20,37,38}$ or enhanced virulence of the virus depending on the site of mutation. ${ }^{38-42}$

Aspartate to glycine mutation (D614G) of the S protein was thought to be 10-times more virulent than the original strain from China (Wuhan-1). ${ }^{38,39}$ Patients infected by the D614G mutant variant had advanced viral loads, ${ }^{43}$ and the overall disease progress was severe when compared with original China (Wuhan-1) strain-infected patients. ${ }^{22}$ The possible explanation might be related to the higher affinity D614G mutant strain for ACE2 than the original China (Wuhan-1) strain. ${ }^{44}$

Furthermore, the potential electro-static change from polar-aspartate to non-polar-glycine on the surface of D614G mutant S protein creates a favorable environment for mutant variants in a hydrophobic pocket of the $\mathrm{S}$ protein. Moreover, the change from large-aspartate residue to small glycine residue on the D614G mutant variant might increase the flexibility of a smooth switchover from the inactive DOWN state to the active UP state. ${ }^{21,39,41}$ Additionally, D614G facilitates cleavage of the spike protein by host proteases and thereby enhances membrane fusion, which is critical for viral entry into host cells. ${ }^{39,42}$

Similar to D614G, V367F, W436R, and D364Y are three other mutant variants of $\mathrm{S}$ protein emerging in Wuhan, Shenzhen, Hong Kong, and France. These variants have 
enhanced structural stabilization of the RBD beta-sheet scaffold and also displayed a higher affinity for human ACE2 just like to D614G variant of the $\mathrm{S}$ protein. ${ }^{40}$

In contrast to D614G and other mutations which enhance infectivity of the virus, there are also mutations which reduce it, such as glycosylation mutants (N331 and N343) mediating protein folding and stability, and amino acid mutation in RBD (R408I). Glycosylation and RBD mutants together lead to drastically reduced viral infectivity. $^{20,45,46}$

R408I mutation seems to disrupt the 408R-glycan hydrogen bond by changing hydrophilic arginine residue with hydrophobic isoleucine residue with no hydrogenbond potential. As evidence for reduced viral infectivity, potentially reduced ACE2 binding affinity which led to reduced virulence of the virus was observed with the R408I mutant strain identified in India. ${ }^{20}$

\section{Effect of Mutant RdRp on Gene Mutation and Therapeutic Target}

ViralRdRp, also known as NSP12, is the complex protein with multiple domains that performs viral RNA synthesis and genome duplication. ${ }^{47}$ An RdRp mutation, particularly in position 14408, makes a great contribution to overall increased viral genome mutation rates. There are also reports on the mutation of $\mathrm{P} 323 \mathrm{~L}$ in $\mathrm{RdRp},{ }^{13,30}$ which could resulted in structural rigidity of the protein and consequently lower replication fidelity of RdRp. ${ }^{31}$

Lower fidelity of RdRp also leads to higher mutation rates of the virus within host cells, which in turn allows new virulent forms to emerge and influence disease profile. $^{31,48}$ Moreover, mutant SARS COV-2 at P323L in RdRp has made infected COVID-19 patients to develop much more severe symptoms. ${ }^{22}$

In addition to its contribution to increased viral genomic mutation rates, mutant RdRp might affect the efficacy of antiviral drugs and thereby disturb therapeutic targets. Antiviral drugs such as remdesivir via their active component (adenosine nucleotide analog) binding to the active sites of RdRpand are being considered potential therapeutic options through viral replication inhibition. ${ }^{27,47,49}$ The active component of remdesivir binds to the RdRp catalytic site and halts nucleic acid elongation. Binding affinity with remdesivir profoundly decreased in several mutants, particularly F480L and V557L variants, strongly suggesting drug resistance. ${ }^{53-55}$
Mutation of RdRpat A97V, A185V and P323 causes alteration of the secondary structure of RdRp. In mutation of RdRp at $\mathrm{A} 97 \mathrm{~V}$ and $\mathrm{A} 185 \mathrm{~V}$, valine (with a relatively larger side chain than alanine) substitutes the alanine (with a relatively smaller side chain than valine) amino acid. In mutation of RdRp at P323L, the proline (with a bulky pyrrolidine ring side chain) substitutes by leucine that might result in loss of the structural integrity of the protein. ${ }^{47,48}$

Recent studies on virtual molecular docking identified many drugs as SARS-CoV-2 RdRp inhibitors, including Simeprevir, Filibuvir, andTegobuvir. ${ }^{49,50}$ The P323L and L329I mutations of RdRp were identified very close to the docking site, probably interfering with the interaction of these drugs with RdRp. ${ }^{4,49-53}$

\section{Conclusion}

The causative agent of the COVID-19 pandemic is prone to a high mutation rate, which leads to changes in the properties of the virus, including its virulence or infectivity, and resistance to antiviral drugs. For instance, mutated SARS COV-2 in S protein at D614G infects ACE2 expressing cells more efficiently, and these boost its virulence. Thus, a patient infected by a mutant variant has higher viral loads and develops more severe symptoms of COVID-19.

Mutated SARS COV-2 at RdRp regions causes low fidelity of $\mathrm{RdRp}$, resulting in higher mutation rates, which in turn lead to the emergence of new virulent strains, influence overall disease profile and interfere with RdRp-targeting pharmacological agents.

Even though a number of genome sequences of this virus have been identified and published, by no means all countries have sequenced the viral genome as yet, information which is important for recognizing mutated genes and newly emerging virus properties.

\section{Copyright/Ethical Concerns}

Since this is a review article, there are no ethical concerns related to it.

\section{Disclosure}

The authors report no conflicts of interest in this work.

\section{References}

1. Ma X, Ph D, Wang D, et al. A novel coronavirus from patients with pneumonia in China, 2019. New Engl J Med Br. 2020;382(8):727-733. doi:10.1056/NEJMoa2001017 
2. Ruan S. Likelihood of survival of coronavirus disease 2019 Scientific and ethical basis for social-distancing interventions against COVID-19. Lancet Infect Dis. 2020;20(6):630-631. doi:10.1016/ S1473-3099(20)30257-7.

3. Guan W, Liang W, Ou C, Du B. Clinical characteristics of coronavirus disease 2019 in China. New Engl J Med Orig. 2020;382 (18):1-14.

4. Wang D, Bo $\mathrm{H}$, Chang $\mathrm{H}$, et al. Clinical characteristics of 138 hospitalized patients with 2019 novel coronavirus-infected pneumonia in Wuhan, China. JAMA. 2020;323(11):1061-1069. doi:10.1001/ jama.2020.1585

5. Wang C, Horby PW, Hayden FG, Gao GF. A novel coronavirus outbreak of global health concern. Lancet. 2020;395(10223):15-18. doi:10.1016/S0140-6736(20)30185-9

6. Koyama T, Platt D, Parida L. Variant analysis of SARS-CoV-2 genomes. Bull World Heal Organ. 2020;2(June):495-504. doi:10.24 71/BLT.20.253591

7. World Health Organization. Infection prevention and control during health care when COVID-19 is suspected. Interim Guid. 2020; (March):1-5.

8. World Health Organization. COVID-19 weekly epidemiological update; 2020 (November).

9. Richardson S, Hirsch JS, Narasimhan M, Crawford JM, Mcginn T, Davidson KW. Presenting characteristics, comorbidities, and outcomes among 5700 patients hospitalized with COVID-19 in the New York City Area. JAMA. 2020;10022(20):2052-2059. doi:10.10 01/jama.2020.6775

10. Chen N, Zhou M, Dong X, et al. Epidemiological and clinical characteristics of 99 cases of 2019 novel coronavirus pneumonia in Wuhan, China: a descriptive study. Lancet. 2020;395(10223): 507-513. doi:10.1016/S0140-6736(20)30211-7

11. Yang X, Yu Y, Xu J, et al. Clinical course and outcomes of critically ill patients with SARS-CoV-2 pneumonia in Wuhan, China: a single-centered, retrospective, observational study. Lancet Respir. 2020;8(5):475-481. doi:10.1016/S2213-2600(20)30079-5

12. Toyoshima Y, Nemoto K, Matsumoto S, Nakamura Y, Kiyotani K. SARS-CoV-2 genomic variations associated with mortality rate of. J Hum Genet. 2020;65(7):1075-1082. doi:10.1038/s10038-0200808-9.

13. Pachetti M, Marini B, Benedetti F, et al. Emerging SARS - CoV - 2 mutation hot spots include a novel RNA - dependent - RNA polymerase variant. J Transl Med. 2020;18(1):1-9. doi:10.1186/s12967020-02344-6

14. Mousavizadeh L, Ghasemi S. Genotype and phenotype of COVID-19: their roles in pathogenesis. J Microbiol Immunol Infect. 2020;54(2):4. doi:10.1016/j.jmii.2020.03.022

15. Tao Z, Tian J, Pei Y, Yuan M, Zhang Y, Dai F. A new coronavirus associated with human respiratory disease in China. Nature. 2020;579(March):265-269. doi:10.1038/s41586-020-2008-3

16. Zhou P, Yang X, Wang X, et al. A pneumonia outbreak associated with a new coronavirus of probable bat origin. Nature. 2020;579 (March):270-273. doi:10.1038/s41586-020-2012-7

17. Kaushal N, Gupta Y, Goyal M, Khaiboullina SF. Mutational frequencies of SARS-CoV-2 genome during the beginning months of the outbreak in USA. Pathogens. 2020;565(9):1-16.

18. Huang Y, Yang C, Xu X, Xu W, Liu S. Structural and functional properties of SARS-CoV-2 spike protein: potential antivirus drug development for COVID-19. Acta Pharmacol Sin. 2020;41 (July):1141-1149. doi:10.1038/s41401-020-0485-4

19. Subissi L, Posthuma CC, Collet A, Zevenhoven-dobbe JC, Gorbalenya AE. One severe acute respiratory syndrome coronavirus protein complex integrates processive RNA polymerase and exonuclease activities. Proc Natl Acad Sci USA. 2014;111(E):3900-3909. doi:10.1073/pnas.1323705111
20. Jia Y, Shen G, Zhang Y, et al. Analysis of the mutation dynamics of SARS-CoV-2 reveals the spread history and emergence of RBD mutant with lower ACE2 binding affinity. bioRxiv. 2020;1-17.

21. Laha S, Chakraborty J, Das S, Kanti S. Characterizations of SARS-CoV-2 mutational profile, spike protein stability and viral transmission. elsevier. 2020;85(104445):1-12.

22. Biswas SK, Mudi SR. RNA-dependent RNA polymerase and spike protein mutant variants of SARS-CoV-2 predominate in severely affected COVID-19 patients. 2020;(July):1-15.

23. Phan T. Infection, genetics and evolution genetic diversity and evolution of SARS-CoV-2. Infect Genet Evol. 2020;81(February):104260. doi:10.1016/j.meegid.2020.104260

24. Yoshimoto FK. The proteins of severe acute respiratory syndrome coronavirus-2 (SARS CoV-2 or n-COV19), the cause of COVID-19. Protein J. 2020;39(23):198-216. doi:10.1007/s10930020-09901-4

25. Duffy S. Why are RNA virus mutation rates so damn high? PLOS Biol. 2018;16(8):1-6. doi:10.1371/journal.pbio.3000003

26. Lu R, Zhao X, Li J, et al. Genomic characterisation and epidemiology of 2019 novel coronavirus: implications for virus origins and receptor binding. Lancet. 2020;395(10224):565-574. doi:10.1016/S01406736(20)30251-8

27. Aftab SO, Ghouri MZ, Masood MU, et al. Analysis of SARS-CoV-2 RNA-dependent RNA polymerase as a potential therapeutic drug target using a computational approach. J Transl Med. 2020;18 (275):1-15. doi:10.1186/s12967-020-02439-0

28. Maria A, Sébastien B, Paul G, et al. A recurrent mutation at position 26,340 of SARS-CoV-2 is associated with failure of the E-gene qRT-PCR utilized in a commercial dual-target diagnostic assay. $J$ Clin Microbiol. 2020;2(July):1-18.

29. Tahamtan A, Ardebili A. Real-time RT-PCR in COVID-19 detection: issues affecting the results. Expert Rev Mol Diagn. 2020;00(00):1-2. doi:10.1080/14737159.2020.1757437

30. Rehman S, Mahmood T, Aziz E, Batool R. Identification of novel mutations in SARS-COV-2 isolates from Turkey. Res Sq. 2020;00:1-21.

31. Begum F, Mukherjee D, Das S, et al. Specific mutations in SARS-CoV2 RNA dependent RNA polymerase and helicase alter protein structure, dynamics and thus function: effect on viral RNA replication. bioRxiv. 2020.

32. Khan MI, Khan ZA, Baig MH, et al. Comparative genome analysis of novel coronavirus (SARS-CoV-2) from different geographical locations and the effect of mutations on major target proteins: an in silico insight. PLoS One. 2020;3(september):1-18. doi:10.1371/journal. pone. 0238344

33. Rahman MS, Islam MR, Alam AS, et al. Evolutionary dynamics of SARS-CoV-2 nucleocapsid protein ( $\mathrm{N}$ protein) and its consequences. bioRxiv. 2020;1-39.

34. Benvenuto D, Angeletti S, Giovanetti M, et al. Evolutionary analysis of SARS-CoV-2: how mutation of Non-Structural Protein 6 (NSP6) could affect viral autophagy. J Infect. 2020;81(1):e24-7. doi:10.1016/ j.jinf.2020.03.058

35. Isabel S, Miraglia LG, Gutierrez JM. Evolutionary and structural analyses of SARS - CoV - 2 D614G spike protein mutation now documented worldwide. Sci Rep. 2020;10(14031):1-9. doi:10.1038/ s41598-020-70827-z

36. Watanabe Y, Berndsen ZT, Raghwani J, et al. Vulnerabilities in coronavirus glycan shields despite extensive glycosylation. Nat Commun. 2020:1-10. doi:10.1038/s41467-020-16567-0

37. Saha P, Banerjee AK, Tripathi PP, Srivastava AK, Ray U. A virus that has gone viral: amino acid mutation in $\mathrm{S}$ protein of Indian isolate of Coronavirus COVID-19 might impact receptor binding, and thus, infectivity. Portl Press. 2020;19:1-8. 
38. Li Q, Wu J, Nie J, et al. The impact of mutations in SARS-CoV-2 spike on viral infectivity and antigenicity 1111 article the impact of mutations in SARS-CoV-2 spike on viral infectivity and antigenicity. Cell Press. 2020;182(9):1284-1294.

39. Hu J, He C-L, Gao Q-Z, et al. D614G mutation of SARS-CoV-2 spike protein enhances viral infectivity. bioRxiv. 2020;1-37.

40. Ou J, Zhou Z, Dai R, et al. Emergence of RBD mutations in circulating SARS-CoV-2 strains enhancing the structural stability and human ACE2 receptor affinity of the spike protein JunxianOu. bioRxiv. 2020;1-30.

41. Volz E, Hill V, McCrone JT, et al. Evaluating the effects of SARS-CoV-2 Spike mutation D614G on transmissibility and pathogenicity. medRxiv. 2020;44:1-41.

42. Eaaswarkhanth M, Al MA, Al-mulla F. Could the D614G substitution in the SARS-CoV-2 spike (S) protein be associated with higher COVID-19 mortality? Int J Infect Dis. 2020;96:459-460. doi:10.1016/j.ijid.2020.05.071

43. Korber B, Wm F, Gnanakaran S, et al. Spike mutation pipeline reveals the emergence of a more transmissible form of SARS-CoV2 Introduction. bioRxiv. 2020;(April):1-33.

44. Zhang L, Jackson CB, Mou H, Ojha A, Rangarajan ES. The D614G mutation in the SARS-CoV-2 spike protein reduces $\mathrm{S} 1$ shedding and increases infectivity. bioRxiv. 2020.

45. Wang L, Wang L, Zhuang H. Pro fi ling and characterization of SARS-CoV-2 mutants , infectivity and antigenicity. Signal Transduct Target Ther. 2020;(August):1-2. doi:10.1038/s41392-02000302-8

46. Xu W, Wang M, Yu D, Zhang X. Variations in SARS-CoV-2 spike protein cell epitopes and glycosylation profiles during global transmission course of COVID-19. Front Immunol. 2020;11 (September):1-12. doi:10.3389/fimmu.2020.565278

47. Chand GB, Banerjee A, Azad GK. Identi fi cation of novel mutations in RNA-dependent RNA polymerases of SARS-CoV-2 and their implications on its protein structure. PeerJ. 2020;8(9492):1-11. doi:10.7717/peerj.9492

48. Karakülah G, Suner A. RdRp mutations are associated with SARS-CoV-2 genome evolution. PeerJ. 2020;8(9587):1-14.
49. Wang Y, Anirudhan V, Du R, Cui Q, Rong L. RNA-dependent RNA polymerase of SARS-CoV-2 as a therapeutic target. J Med Virol. 2020;(July):1-11.

50. Ruan Z, Liu C, Guo Y, He Z, Huang X, Jia X. Potential inhibitors targeting RNA-dependent RNA polymerase activity (NSP12) of SARS-CoV-2. NOT PEER-REVIEWED. 2020;(March):1-16.

51. Goldhill DH, Aartjan JW, Fletcher RA, Langat P, Zambon M, Lackenby A. The mechanism of resistance to favipiravir in influenza. Proc Natl Acad Sci U S A. 2018;115(45):11613-11618. doi:10.1073/pnas.1811345115

52. Delang L, Froeyen M, Herdewijn P, Neyts J. Identification of a novel resistance mutation for benzimidazole inhibitors of the $\mathrm{HCV}$ RNA-dependent RNA polymerase. Antiviral Res. 2012;93(1):30-38. doi:10.1016/j.antiviral.2011.10.012

53. Badua CL. Genomic and proteomic mutation landscapes of SARSCoV-2.pdf. Wiley. 2020;1(september):1-20.

54. Padhi AK, Shukla R, Tripathi T. Rational design of the remdesivir binding site in the RNA-dependent RNA polymerase of SARS-CoV2: implications for potential resistance. bioRxiv. 2020;1-26.

55. Agostini ML, Andres EL, Sims AC, et al. Coronavirus susceptibility to the antiviral remdesivir(GS- 5734) is mediated by the viral polymerase and the. Am Soc Microbiol. 2018;9(2):1-15.

56. Holland LA, Kaelin EA, Maqsood R, et al. An 81-nucleotide deletion in SARS-CoV-2 ORF7a identified from sentinel surveillance in Arizona (January to March 2020). J Virol. 2020;94(14). doi:10.1128/JVI.00711-20.

57. Bal A, Destras G, Gaymard A, et al. Molecular characterization of SARS-CoV-2 in the first COVID-19 cluster in France reveals an amino acid deletion in nsp2 (Asp268del). Clin Microbiol Infect. 2020;26(7):960-962. doi:10.1016/j.cmi.2020.03.020

58. Benedetti F, Snyder GA, Giovanetti M, et al. Emerging of a SARS-CoV-2 viral strain with a deletion in nsp1. J Transl Med. 2020;18(1). doi:10.1186/s12967-020-02507-5.
Infection and Drug Resistance

\section{Publish your work in this journal}

Infection and Drug Resistance is an international, peer-reviewed openaccess journal that focuses on the optimal treatment of infection (bacterial, fungal and viral) and the development and institution of preventive strategies to minimize the development and spread of resistance. The journal is specifically concerned with the epidemiology of antibiotic resistance and the mechanisms of resistance development and diffusion in both hospitals and the community. The manuscript management system is completely online and includes a very quick and fair peerreview system, which is all easy to use. Visit http://www.dovepress.com/ testimonials.php to read real quotes from published authors. 